\title{
Primary trimethylaminuria (fish odor syndrome) and hypothyroidism in an adolescent
}

\author{
Mustafa Kılıç \\ Division of Metabolism, Dr. Sami Ulus Maternity and Children's Health and Diseases Training and Research Hospital, \\ Ankara, Turkey.E-mail: kilickorkmaz@yahoo.com.tr \\ Received: 21st December 2016, Revised: 27th February 2017, Accepted: 28th February 2017
}

SUMMARY: Kılıç M. Primary trimethylaminuria (fish odor syndrome) and hypothyroidism in an adolescent. Turk J Pediatr 2017; 59: 614-616.

Primary trimethylaminuria (fish-odor syndrome) is a rare autosomal recessive inherited metabolic disorder due to decreased metabolism of trimethylamine by enzyme flavin-containing monooxygenase 3 . We report an adolescent boy who was socially distressed with malodor and diagnosed trimethylaminuria by molecular analyses. The patient was previously found to have primary hypothyroidism at another center when he was being investigated for malodor. Our aim is to remind awareness of this rare, socially devastating metabolic disorder to physicians, especially family doctors and specialists such as pediatricians, adolescent doctors, psychiatrists and dermatologists. We also searched for a possible association between trimethylaminuria and hypothyroidism but didn't find out an exact pathophysiological link. Coexistence of primary trimethylaminuria and hypothyroidism might be coincidental.

Key words: trimethylaminuria, fish odor syndrome, malodor, FMO3, hypothyroidism.

Trimethylaminuria is a rare, socially debilitating metabolic disorder due to decreased metabolism of trimethylamine in liver by enzyme flavincontaining monooxygenase 3 (FMO3) secondary to FMO3 gene defect. ${ }^{1-4}$ Affected patients have episodic or persistent unpleasant odor and also their breath, sweat, urine, saliva and other secretions smell characteristic of rotting fish. ${ }^{5}$ Hypertension, migraine and other tyramine reactions are also described in these patients. ${ }^{1,3}$ Psychiatric counseling is also important as it has strong psychosocial effects. We report one adolescent boy who was socially distressed with malodor and was diagnosed with primary trimethylaminuria. He was previously found to have hypothyroidism at another center when he was being investigated for malodor. We also analyzed if primary trimethylaminuria and primary hypothyroidism is associated or their coexistence is coincidental.

\section{Case Report}

A twelve-year-old Turkish adolescent boy was admitted to hospital for the malodor of urine and sweat which had been noticed at six years of age. The complaint of patient was increasing by meat consumption. Urine and sweat was in normal color but smelled unpleasant. Parents had distant kinship. He was healthy and had normal diet. There was a history of levothyroxine treatment for hypothyroidism starting at six-years-old and varicocele operation at 11years of age. Hypothyroidism was diagnosed at another center when he was being investigated for malodor. At that time TSH level was $17 \mu \mathrm{IU} /$ $\mathrm{ml}(\mathrm{N}: 0.6-5.5)$, free T4 level was $0.89 \mathrm{ng} / \mathrm{dl}$ (0.8-1.9) and levothyroxine $50 \mu \mathrm{g} /$ day $(2 \mu \mathrm{g} /$ $\mathrm{kg}$ /day) was started. His mother had a goiter and his sister also had hypothyroidism. Since the etiology of malodor was not detected, he was referred to our center. Physical examination findings were normal. Laboratory findings (total blood count, liver and kidney function tests, urine analyses and culture, tandem mass, urine organic acid, blood and urine quantitative amino acid analyses, biotinidase activity, homocysteine, lactate, pyruvate) were unremarkable except for the mild TSH elevation (7.38 $\mu \mathrm{IU} / \mathrm{ml}, \mathrm{N}:$ 0.6-5.5; free T4 $1.23 \mathrm{ng} / \mathrm{dl}, \mathrm{N}$ : 0.8-1.9) and low vitamin B12 levels (139 pg/ml, $\mathrm{N}:$ 200-1090). Thyroid antibodies (anti-thyroid peroxidase and anti-thyrogloblin) were negative. 
Thyroid sonography was normal. Unfortunately, urine iodine was not analyzed. Iodine deficiency and/or dyshormonogenesis were thought as possible causes of primary hypothyroidism. Thyroxine treatment has been continued with increasing dosage. Subclinical vitamin B12 deficiency without hematological changes and neurological impairment was observed and vitamin B12 treatment was started. After eliminating the common causes of malodor, primary trimethylaminuria was suspected. Direct sequencing of FMO3 gene revealed a homozygous c.1160G >A;p.R387H mutation in exon 7 and confirmed the diagnosis. Choline restricted diet, usage of acidic soap, and taking frequent shower were advised. In condition of heavy smell, oral metronidazole $(2 \times 500 \mathrm{mg})$ treatment daily for two weeks was suggested. $\mathrm{He}$ and his family relieved after the diagnosis and his symptoms decreased after treatment. We thank the family of the patient for their participation involving informed consent.

\section{Discussion}

Fewer than 300 trimethylaminuria patients with more than 30 different mutations and over 40 polymorphisms are described in the literature. ${ }^{4,6,7}$ It is rare disorder but exact prevalence is not known. However, it is much higher than it is expected. ${ }^{8}$ In our patient we couldn't calculate the percentage of TMAO in the patient's urine but we made molecular analyses of FMO3 gene for the diagnosis. An already known c.1160G >A;p. R387H homozygous mutation was detected. ${ }^{1}$ Although, it has been well documented in several populations, we did not find any Turkish patient with trimethylaminuria reported in the literature.

A few metabolic disorders are related to psychiatric disorders. Phenylketonuria, homocystinuria, cobalamin defects, NiemannPick type C, mucopolysaccharidosis type III, creatine synthesis and transport defects, disorders of purine and pyrimidine metabolism and trimethylaminuria are well known examples. Attention deficit hyperactivity syndrome, autism spectrum disorders, schizophrenia, dementia, anxiety and mood disorders are some of the metabolic disease related psychiatric symptoms. Trimethylaminuria related psychosocial problems such as low self-esteem, social isolation, anxiety, depression, paranoia, low school and academic performance and even suicide attempts are well described in the literature. ${ }^{8,9}$ Adolescence period, especially middle adolescence, is important for their development with its own psychological effects, in which any physical or emotional impairment can cause major problems especially at this stage. Anxiety and distress was also observed in our patient and he relieved after diagnosis and treatment. Hence we prevented the development of other psycho-social problems in our patient by making an early diagnosis and treatment. Furthermore, if we haven't made the correct diagnoses, it could have been considered as a primary psychological disorder. Therefore, it is important to find out primary pathologic defect before psychiatric diagnosis and consultation.

Inborn errors of amino acid metabolism associated with peculiar odor are described in glutaric aciduria type II and isovaleric acidemia (sweaty feet), hawkinsinuria (swimming pool), 3-hydroxy-3-methylglutaric aciduria (cat urine), multiple carboxilase deficiency (tomcat urine), maple syrup urine disease (maple syrup), hypermethioninemia and tyrosinemia (boiled cabbage), phenylketonuria (mousey or musty) and trimethylaminuria (rotting fish). It is also important to eliminate other common causes of fish odor, such as poor hygiene, gingivitis, bacterial vaginosis, urinary tract infections and chronic and advanced liver and kidney disease. ${ }^{8}$ Physical examination and laboratory investigation including metabolic screening were normal for the other causes of malodor and fish odor in our patient.

There are many reports discussing trimethylamine and human health consequences and it is a novel research topic nowadays. A number of diseases are associated with abnormal levels of TMA, including renal disorders, cancer, obesity, diabetes, cardiovascular diseases and neuropsychiatric disorders. ${ }^{10}$ There is one animal study that discussed the trimethylamine and thyroid function, in there the measurements of thyroid activity and hepatic TMA oxidase activity in domestic fowls were studied and results indicated that the short-term depression of TMA oxidation in birds sensitive to rapeseed meal is not mediated by the thyroid. ${ }^{11}$ We searched for 
an association between trimethylaminuria and hypothyroidism but there are no reports in the literature that links two disorders, and it is more likely coincidental. Only one trimethylaminuria patient with hypothyroidism had been reported. ${ }^{12}$ In our patient, since clinical features appeared at the same time and both diseases are related to monooxygenase system, we supposed that thyroid hormone synthesis especially in the level of iodine oxidation with thyroid peroxidase might be affected secondary to monooxygenase system. To date, only two trimethylaminuria cases including this case had concomitant hypothyroidism, it is difficult to say that there is a real pathophysiological link.

In conclusion molecular genetic analysis should be considered in patients with suspicion of trimethylaminuria. Many basic precautions and treatments can save these psychiatric difficulties and relax the patient and his family. There is no stated exact role of FMO3 in thyroid hormone biogenesis. However further studies and cases are needed to investigate this phenomenon in greater depth.

\section{REFERENCES}

1. Akerman BR, Lemass H, Chow LM, et al. Trimethylaminuria is caused by mutations of the FMO3 gene in a North American Cohort. Mol Genet Metab 1999; 68: 24-31.

2. Christodoulou J. Trimethylaminuria: An underrecognised and socially debilitating metabolic disorder. J Paediatr Child Health 2012; 48: 153-155.

3. Treacy EP, Akerman BR, Chow LM, et al. Mutations of the flavin-containing monooxygenase gene (FMO3) cause trimethylaminuria, a defect in detoxication. Hum Mol Genet 1998; 7: 839-845.

4. Dolphin CT, Janmohamed A, Smith RL, Shephard EA, Phillips IR. Missense mutation in flavin-containing mono-oxygenase 3 gene, FMO3, underlies fish-odour syndrome. Nat Genet 1997; 17: 491-494.

5. Ulman CA, Trevino JJ, Miller M, Gandhi RK. Fish odor syndrome: A case report of trimethylaminuria. Dermatol Online J 2014; 20: 21260.

6. Shephard EA, Treacy EP, Phillips IR. Clinical utility gene card for: Trimethylaminuria-update 2014. Eur J Hum Genet 2015; 23. doi: 10.1038/ejhg.2014.226.

7. Motika MS, Zhang J, Zheng X, Riedler K, Cashman JR. Novel variants of the human flavin-containing monooxygenase 3 (FMO3) gene associated with trimethylaminuria. Mol Genet Metab 2009; 97: 128135.

8. Rehman HU. Fish odor syndrome. Postgrad Med J 1999; 75: 451-452.
9. Messenger J, Clark S, Massick S, Bechtel M. A review of trimethylaminuria (fish odor syndrome). J Clin Aesthet Dermatol 2013; 6: 45-48.

10. Goel JC, Gaur A, Singhal V, Parakh N, Bhargava B, Sharma A. The complex metabolism of trimethylamine in humans: endogenous and exogenous sourcesCORRIGENDUM. Expert Rev Mol Med 2016; 18: e19.

11. Davison TF, Pearson AW, Rea J, Greenwood NM. Thyroid function in relation to trimethylamine oxidation in immature domestic fowl fed on rapeseed meal. $\mathrm{Br}$ Poult Sci 1981; 22: 437-443.

12. Li M, Al-Sarraf A, Sinclair G, Frohlich J. Fish odour syndrome. CMAJ 2011; 183: 929-932. 